\title{
Locking of neoclassical tearing modes by error fields and its stabilization by RF current
}

\author{
Q. Yu and S. Günter
}

Max-Planck-Institut für Plasmaphysik, EURATOM Association, 85748 Garching, Germany

The locking of neoclassical tearing modes (NTMs) by error fields is studied numerically. In the regime with low mode frequency and large plasma viscosity, the required field amplitude for mode locking is found to be proportional to the plasma viscosity and the mode frequency but inversely proportional to the square of the magnetic island width and the Alfven velocity, being similar to that of the classical tearing mode. This indicates that NTMs will be locked to low amplitude error fields in a fusion reactor. The stabilization of NTMs by $\mathrm{RF}$ current in the presence of a static helical field is therefore further investigated. The applied helical field allows to control the location of the island's o-point to be in the RF wave deposition region, to enable the NTM stabilization by RF current after mode locking. When the island is large enough to be locked by a small amplitude helical field in the desired phase, the island is reduced to a smaller width by RF current compared to the case without the helical field. This suggests a possible way to enhance the stabilization of NTMs by RF current.

PACS: 52.35.Py, 52.35.Vd, 52.55.Tn, 52.35.-g 


\section{Introduction}

Neoclassical tearing modes (NTMs) are found to limit the tokamak plasma pressure well below the prediction of ideal magnetohydrodynamic (MHD) calculations for positive magnetic shear [1-7]. In recent years much effort has been devoted to NTMs, to understand the threshold for their onset and their effect on plasma performance [1-16]. Compared to the $\mathrm{m} / \mathrm{n}=3 / 2$ mode or modes with higher mode numbers, the $2 / 1 \mathrm{NTM}$ often leads to a stronger degradation of plasma confinement or even to mode locking and disruption once the mode amplitude is sufficiently large. Therefore, an active stabilization of NTMs is required for a fusion reactor.

A straightforward method for stabilizing NTMs is to use localized RF current drive, to fill the current hole caused by the disappearance of the bootstrap current inside the island. Due to its localized deposition, Electron Cyclotron Current Drive (ECCD) is very appropriate for this stabilization purpose without causing a significant change of the overall plasma current density profile. It has been shown in tokamak experiments that localized ECCD can stabilize NTMs as predicted by theoretical results [17-26]. In these experiments the rotating magnetic island passes through the RF wave deposition region periodically when the radial wave deposition is around the rational surface, and both modulated or non-modulated ECCD can generate RF current around the island's o-point, being the effective current for mode stabilization [22,24,25]. Even large amplitude rotating 2/1 NTMs can be stabilized if the RF current around the island's o-point is sufficiently large $[19,20]$.

On the other hand it is well known that the intrinsic error field of tokamaks or externally applied resonant magnetic perturbations can have a significant effect on magnetic islands [27-39]. Once the island is large enough, it will be locked to the error field. Even if the error field amplitude is sufficiently small, the helical current induced in the vacuum vessel by an unstable mode will produce a helical field to act back on the island, leading to the mode locking of a large island [27-29]. After the island's o-point is locked at a particular toroidal and poloidal angle by the error field, being not necessarily covered by the RF waves 
deposition region, it becomes impossible to stabilize the NTM by RF current. Therefore, it is important to understand the threshold for NTM locking, in particular as locked 2/1 modes often lead to major plasma disruptions $[27,28]$.

Most recent studies on the error field focused on the field penetration threshold. The penetration threshold was found to be very small, typically $b_{\mathrm{ra}} / \mathrm{B}_{\mathrm{t}} \sim 10^{-4}-10^{-3}$ on existing tokamaks, where $b_{r a}$ is the radial component of the error field at the plasma edge $r=a$, and $B_{t}$ is the toroidal magnetic field [33-39]. The new experimental results indicated the important role of the electron diamagnetic drift $[38,39]$. The penetration threshold has a minimum when the applied helical field frequency is the same as the mode frequency being determined by both the plasma rotation and the diamagnetic drift. As the field frequency deviates from the mode frequency, the threshold significantly increases and is asymmetry on the two sides of the minimum $[38,39]$. The nonlinear numerical results based on two fluids equations agree with the experimental results, and the asymmetry is found to be resulted from the change of the diamagnetic drift frequency due to parallel transport [40]. These latest results provide new insight into the mode penetration physics and could lead to a more reasonable prediction of the mode penetration threshold of a fusion reactor.

Similar to mode penetration, the mode locking of NTMs is also an important issue for a fusion reactor. The required field amplitude for mode locking should be even smaller than that for mode penetration, since the electromagnetic force to stop the island rotation is proportional to the amplitude of NTMs [27-32]. It will be shown in the present paper that the mode locking threshold is proportional to the mode frequency but inversely proportional to the square of the Alfven velocity. Such a scaling indicates that low-m NTMs will be much more easily locked by the error field in a fusion reactor than in existing tokamaks.

In the first part of this paper the locking of NTMs by an error field is studied numerically using large aspect-ratio tokamak approximation, focusing on the dependence of the mode locking threshold on plasma parameters. Both the parallel and the perpendicular heat transport are self-consistently included in our calculations to take into account the 
bootstrap current perturbation, which is essential for modelling NTMs. It is found that NTMs will be locked at a small amplitude by the error field in a reactor plasma. This means that in order to stabilize a large amplitude NTM by RF current, additional measures are needed either to maintain the island rotation or to control the phase of the locked island according to the RF wave deposition.

This fact motivates the second part of the present work: the stabilization of NTMs by RF current in the presence of an applied static helical field. The actively applied field allows to control the location of the island's o-point to be in the RF wave deposition region, to enable the NTM stabilization by RF current after mode locking. Although the helical field has a destabilizing effect on the NTM, at given RF power the effective RF current for mode stabilization, the RF current around the island's o-point, is two times larger than that of a continuous or modulated RF current drive for a rotating NTM. The presented numerical results show that the NTM is reduced to a smaller amplitude by the larger RF current in this case compared to that without the helical field, if the island is large enough to be locked by a small amplitude helical field in the desired phase. Such an enhanced stabilizing efficiency of the RF current is expected to be useful for the stabilization of NTMs, especially of the $2 / 1$ NTM in a fusion reactor.

In Section 2 our numerical results on the locking of NTMs by a helical field are presented. The stabilization of NTMs by the RF current in the presence of a helical field is described in Section 3, and the summary is given in Section 4.

\section{Locking of NTMs by error fields}

\subsection{The model}

The large aspect-ratio tokamak approximation is utilized here. The magnetic field is defined as $\mathbf{B}=\mathrm{B}_{\mathrm{t}} \mathbf{e}_{\mathrm{t}}-(\mathrm{kr} / \mathrm{m}) \mathrm{B}_{\mathrm{t}} \mathbf{e}_{\theta}+\nabla \psi \times \mathbf{e}_{\mathrm{t}}$, where $\psi$ is the helical flux function, $\mathrm{m} / \mathrm{r}$ and $\mathrm{k}=\mathrm{n} / \mathrm{R}$ are the wave vectors in $\mathbf{e}_{\theta}$ (poloidal) and $\mathbf{e}_{\mathrm{t}}$ (toroidal) direction, respectively, $\mathrm{R}$ is the major radius, and $\mathrm{m}$ and $\mathrm{n}$ are the poloidal and toroidal mode numbers. 
The basic equations utilized here are Ohm's law, the equation of motion and the energy conservation equation. Normalizing the length to the minor radius a, the time $\mathrm{t}$ to the resistive time $\tau_{R}=a^{2} \mu_{0} / \eta$, the helical flux $\psi$ to $\mathrm{aB}_{\mathrm{t}}, \mathbf{v}$ to $\mathrm{a} / \tau_{\mathrm{R}}$, and the electron temperature $\mathrm{T}_{\mathrm{e}}$ to its value at the magnetic axis, theses equations become

$$
\begin{aligned}
& \frac{d \psi}{d t}=E-\eta\left(j-j_{b}\right) \\
& \frac{d U}{d t}=-S^{2} \nabla_{\|} j+\mu \nabla_{\perp}^{2} U+S_{m} \\
& \frac{3}{2} n_{e} \frac{d T_{e}}{d t}=n_{e} \nabla_{\|}\left(\chi_{\|} \nabla T_{e}\right)+n_{e} \nabla_{\perp}\left(\chi_{\perp} \nabla T_{e}\right)+S_{p}
\end{aligned}
$$

where $d / d t=\partial / \partial t+v_{\perp} \cdot \nabla, j=\nabla_{\perp}^{2} \psi-2 n B_{t} /(m R)$ and $j_{b}=-c_{b}(r / R)^{1 / 2} n_{e} T_{e}^{\prime} / B_{\theta}$ being the toroidal plasma current density and the bootstrap current density, respectively. $\mathrm{B}_{\theta}$ is the poloidal magnetic field, $c_{b}$ is a constant of the order of unity, $n_{e}$ is the electron density, $\eta$ is the normalized plasma resistivity, and $\mathrm{E}$ is the equilibrium electric field for maintaining the equilibrium plasma current. The magnetic Reynolds number $S=\tau_{R} / \tau_{A}$, where $\tau_{A}=a / V_{A}$ being the toroidal Alfven time. $U=-\nabla_{\perp}^{2} \phi$ being the plasma vorticity, and $\mu$ is the plasma viscosity. $\chi_{\|}$and $\chi_{\perp}$ are the parallel and perpendicular heat conductivities, $S_{p}$ is the heating power, and $S_{m}$ in equation (2) is the poloidal momentum source which leads to an equilibrium plasma rotation.

Equations (1)-(3) provide a simple model for modelling the nonlinear evolution of NTMs [41], which reduce to the MHD equations if $j_{b}=0$ is taken in Ohm's law to neglect the bootstrap current. The plasma density and the ion temperature are assumed to be constant for simplicity. This greatly reduces the computation time compared to the case of solving two fluids equations directly [40]. From the physics point of view this approximation is reasonable, since for a sufficiently large magnetic island the plasma density and ion temperature profiles become flattening in the island region similar to the electron temperature profile. In this case the local diamagnetic drift frequency is nearly zero, and the bootstrap current perturbation results from the change of plasma density is similar to that of plasma temperature. While for the mode penetration being relevant to the small island, the difference 
between the heat and particle transport is significant. The plasma density needs to be treated separately in order to calculate the change of the electron diamagnetic drift frequency, which is important in determining the penetration threshold [40].

The effect of the error field is taken into account by the boundary condition

$$
\psi_{\mathrm{m} / \mathrm{n}}(\mathrm{r}=\mathrm{a})=\psi_{\mathrm{a}} \mathrm{aB} \mathrm{B}_{\mathrm{t}} \cos (\mathrm{m} \theta+\mathrm{n} \phi)
$$

where $\psi_{\mathrm{a}}$ describes the normalized error field amplitude of the $\mathrm{m} / \mathrm{n}$ component at $\mathrm{r}=\mathrm{a}$. The radial error field at $r=a$ is given by $b_{r a}=m \psi_{a} B_{t}$.

A monotonic profile for the safety factor $q$ is assumed with the $q=3 / 2$ surface located at $r_{s}=0.575 a$ and the local magnetic shear length $L_{q}=q /\left(a q^{\prime}\right)=0.57$, based on ASDEX-Upgrade parameters. Most calculations are done for the $3 / 2$ mode. For vanishing bootstrap current perturbation the plasma is stable against $\mathrm{m} / \mathrm{n}=3 / 2$ (classical) tearing modes.

\subsection{Numerical results}

Equations (1)-(3) are solved simultaneously using the initial value code TM1, which has been used for modelling the nonlinear growth and saturation of NTMs and their stabilization by RF current earlier [25,41]. Dedicated numerical methods are utilized in the code to keep the numerical error at very low level even for high values of $S$ and $\chi_{\|} / \chi_{\perp}[41-43]$.

Figure 1 demonstrates the nonlinear growth and saturation of a $m / n=3 / 2$ magnetic island driven by the bootstrap current, showing the normalized island width w/a as a function of the normalized time $t / \tau_{\mathrm{R}}$. The input parameters are as follows: the magnetic Reynolds number $\mathrm{S}=10^{8}$, the viscous time $\tau_{\mu}=\mathrm{a}^{2} / \mu=0.1 \tau_{\mathrm{R}}, \chi_{\perp}=30 \mathrm{a}^{2} / \tau_{\mathrm{R}}$, and $\chi_{\|}=3.0 \times 10^{10} \mathrm{a}^{2} / \tau_{\mathrm{R}}$. The local equilibrium bootstrap current density fraction $\mathrm{f}_{\mathrm{b}}$ is 12.7 percent of the local plasma current density. These input parameters are also used for the following simulations except mentioned elsewhere. The solid (dashed) curve corresponds to an error field amplitude $\psi_{\mathrm{a}}=5 \times 10^{-8}\left(\psi_{\mathrm{a}}\right.$ $=10^{-7}$ ) of the $\mathrm{m} / \mathrm{n}=3 / 2$ component. The islands grow and saturate with the saturated island widths being only slightly different for these two cases, since the error field amplitude is quite 
small. The island width is dominated by the perturbed bootstrap current and the equilibrium plasma current density profile.

The corresponding time evolution of the mode frequency is shown in figure 2 by the solid (dashed) curve for $\psi_{\mathrm{a}}=5 \times 10^{-8}\left(10^{-7}\right)$. The island rotation results from the poloidal plasma rotation sustained by the momentum source in equation (2). For $\psi_{\mathrm{a}}=5 \times 10^{-8}$ the mode frequency oscillates around a finite value, indicating that the island still rotates. For a larger $\psi_{\mathrm{a}}, \psi_{\mathrm{a}}=10^{-7}$, the island is locked to the error field, so that the mode frequency finally approaches zero. The required field amplitude for mode locking is quite small due to the high S number.

In figure 3 the radial profile of the $3 / 2$ component of the helical flux, $\psi_{3 / 2}$, in steady state is shown for $\psi_{\mathrm{a}}=10^{-7}$, where the solid (dashed) curve represents the real (imaginary) part. The $\psi_{3 / 2}$ profile looks like that of an usual NTM [41]. The much smaller imaginary part compared to the real one indicates a small phase shift between the mode and the error field, being required for the balance between the electromagnetic force exerted by the error field and the friction from the surrounding plasma on the magnetic island.

Corresponding to figure 1 , radial profiles of the $\mathrm{m} / \mathrm{n}=0 / 0$ component of the poloidal plasma velocity in steady state are shown in figure 4 by the solid (dotted) curve for $\psi_{\mathrm{a}}=5 \times 10^{-8}$ $\left(10^{-7}\right)$. For the locked case with $\psi_{\mathrm{a}}=10^{-7}$, the local velocity near the rational surface is brought to zero, while for $\psi_{\mathrm{a}}=5 \times 10^{-8}$ the local velocity oscillates around a finite value, as shown by the two solid curves for $\mathrm{t}=0.09680$ and $0.09681 \tau_{\mathrm{R}}$, respectively. Such an oscillation corresponds to the oscillation of the mode frequency as shown in figure 2. Away from the rational surface the velocity only slightly changes. The dashed curve shows the original equilibrium velocity profile.

The radial profile of the $\mathrm{m} / \mathrm{n}=0 / 0$ component of the electron temperature in steady state is shown in figure 5 by the solid curve for $\psi_{\mathrm{a}}=10^{-7}$. The local temperature gradient reduces to nearly zero around the rational surface due to the fast parallel transport, since the 
island width is sufficiently large [43]. This indicates that the ion polarization current associated with the diamagnetic drift can be neglected for a sufficiently large island. The dotted curve shows the original equilibrium temperature profile. The temperature profile for the case $\psi_{\mathrm{a}}=5 \times 10^{-8}$ is essentially the same as that for $\psi_{\mathrm{a}}=10^{-7}$, since island widths are about the same for these two cases as seen in figure 1.

To study the dependence of the mode locking threshold on plasma parameters, in figure 6 the required $\psi_{\mathrm{a}}$ to lock the island is shown as a function of the magnetic Reynolds number $\mathrm{S}$ in the $\log \left(\psi_{\mathrm{a}}\right) \sim \log (\mathrm{S})$ plane. The solid circles show the $\psi_{\mathrm{a}}$ leading to mode locking, while the empty squares show the $\psi_{\mathrm{a}}$ for which the mode is not locked yet. The mode locking threshold is between these two curves. The required $\psi_{\mathrm{a}}$ to lock the NTM is found to be proportional to $\mathrm{S}^{-2}$. Since the mode locking is due to the electromagnetic force exerted by the error field on the island, this force is proportional to $S^{2}$ when keeping other parameters unchanged as seen from equation (2). Calculations have also been carried out for the same $S$ value but different plasma resistivity, and the obtained mode locking threshold is the same. The locking threshold is therefore proportional to $\mathrm{V}_{\mathrm{A}}{ }^{-2}$ but is independent of the plasma resistivity, being different from the error field penetration.

For obtaining the above and the following results, the momentum source $S_{m}$ in equation (2) is taken to be the form $S_{m} \sim\left[1-(r / a)^{2}\right]^{2}$. Other forms of $S_{m}$, such as $S_{m} \sim\left[1-(r / a)^{2}\right]^{3}$ and $\mathrm{S}_{\mathrm{m}} \sim\left[1-(\mathrm{r} / \mathrm{a})^{2}\right]^{18}$, have also been used for comparison. The obtained results are found to be the same as long as the equilibrium rotation velocity at the rational surface are taken to be the same. This indicates that the detail radial profile of $S_{m}$ has little effect on the locking threshold. For checking numerical convergence, up to 3000 radial grid points and 30 Fourier components have been utilized.

In figure 7 the required $\psi_{\mathrm{a}}$ to lock the island is shown as a function of the normalized island width w/a. The solid circles (empty squares) show the $\psi_{\mathrm{a}}$ for which the mode is locked (not locked). The different saturated island width results from different input values of the 
bootstrap current density fraction $\mathrm{f}_{\mathrm{b}}$. The slope of the curve shows that the required $\psi_{\mathrm{a}}$ for mode locking is proportional to $\mathrm{w}^{-2}$, as the electromagnetic force applied on the magnetic island is proportional to the amplitude of the NTM.

For mode locking, the electromagnetic force to stop the island rotation has to balance the plasma viscous and inertia forces. Figure 8 shows the mode locking threshold, $\log \left(\psi_{\mathrm{a}}\right)$ versus $\log (\mu)$, where $\mu$ is normalized plasma viscosity in the unit of $a^{2} / \tau_{R}$. The solid circles (empty squares) show the $\psi_{\mathrm{a}}$ for which the mode is locked (not locked). The required $\psi_{\mathrm{a}}$ for mode locking is proportional to the plasma viscosity $\mu$ for large $\mu$, but its dependence on $\mu$ is weak for small $\mu$. In the large $\mu$ region the viscous force dominates over plasma inertia, so that the required $\psi_{\mathrm{a}}$ to lock the island is proportional to $\mu$. In the small $\mu$ region the plasma viscous force is not important, and thus the locking threshold changes little with decreasing $\mu$.

Figure 9 shows the mode locking threshold versus the normalized island angular rotation frequency $\omega \tau_{R}$ for two cases, $\tau_{\mu}=0.1 \tau_{R}$ and $\tau_{\mu}=10^{-4} \tau_{R}$. The solid circles (empty squares) show the $\psi_{\mathrm{a}}$ for which the mode is locked (not locked). For $\tau_{\mu}=0.1 \tau_{\mathrm{R}}$ the required $\psi_{\mathrm{a}}$ for mode locking approximately linearly increases with increasing $\omega$ for small $\omega$, while for large $\omega$ the required $\psi_{\mathrm{a}}$ increases faster since plasma inertia becomes more important. For $\tau_{\mu}$ $=10^{-4} \tau_{\mathrm{R}}$ the required $\psi_{\mathrm{a}}$ for mode locking is again proportional to $\omega$, because the effect of plasma inertia is weaker than that of the plasma viscosity in this case.

Calculations have also been carried out for the $m / n=2 / 1$ mode. An example is given in figure 10, in which the mode locking threshold versus $\omega \tau_{\mathrm{R}}$ is shown for three cases: the bootstrap current density fraction $\mathrm{f}_{\mathrm{b}}=0$ and 0.052 for $\tau_{\mu}=10^{-4} \tau_{\mathrm{R}}$, and $\mathrm{f}_{\mathrm{b}}=0.052$ for $\tau_{\mu}=10^{-2} \tau_{\mathrm{R}}$. The saturated island widths are $0.097 \mathrm{a}$ and $0.157 \mathrm{a}$ for $\mathrm{f}_{\mathrm{b}}=0$ and 0.052 , respectively. The solid circles (empty squares) show the $\psi_{\mathrm{a}}$ for which the mode is locked (not locked). It is seen that the required $\psi_{\mathrm{a}}$ for mode locking linearly increases with $\omega$ for a small $\tau_{\mu}$ or $\omega$, similar to what has been found for the $3 / 2$ mode. Same scaling, $\psi_{\mathrm{a}} \sim \omega \tau_{\mathrm{R}}$, is obtained for both the classical 
tearing modes $\left(f_{b}=0\right)$ and the NTM $\left(f_{b}=0.052\right)$. The smaller value of $\psi_{a}$ required to lock the NTM is due to its larger saturated island width.

\subsection{Discussion on mode locking}

For a fusion reactor the plasma rotation velocity is expected to be low, since neutral beam injection (NBI) heating is not needed for a burning plasma. The plasma rotation velocity could be of the order of the diamagnetic drift velocity. The momentum confinement time of tokamak plasmas is usually about the same as the energy confinement time, indicating that the plasma viscosity is anomalous as the heat conductivity. The results obtained in the last section show that, for a sufficiently low plasma rotation frequency and a large viscosity, the required $\psi_{\mathrm{a}}$ for mode locking scales as $\psi_{\mathrm{a}} \sim \mu \omega\left(\mathrm{V}_{\mathrm{A}} \mathrm{W} / \mathrm{a}\right)^{-2}$. Using the numerical results obtained, the mode locking threshold of the $\mathrm{m} / \mathrm{n}=3 / 2$ mode is found to be

$$
\psi_{\mathrm{a}}=75 \mu \omega\left(\mathrm{V}_{\mathrm{A}} \mathrm{w} / \mathrm{a}\right)^{-2}
$$

where $\mu$ is in the unit of $\mathrm{m}^{2} / \mathrm{s}, \mathrm{V}_{\mathrm{A}}$ in $\mathrm{m} / \mathrm{s}$, and $\omega$ in $\mathrm{s}^{-1}$.

Equation (5) results from the reduction of the poloidal island rotation by the poloidal electromagnetic force. In tokamak experiments due to neoclassical effects the plasma rotation is mostly in toroidal direction. This will lead to two modifications [27,28]: (a) The electromagnetic force to slow down the island rotation in the toroidal direction is smaller by a factor $(\mathrm{n} / \mathrm{m})\left(\mathrm{r}_{\mathrm{s}} / \mathrm{R}\right)$ compared to that in the poloidal direction. (b) To have the same mode frequency due the plasma rotation, the toroidal rotation speed should be $(\mathrm{m} / \mathrm{n})\left(\mathrm{R} / \mathrm{r}_{\mathrm{s}}\right)$ times larger than the poloidal one. These two effects lead to a larger ratio of the viscous force to the electromagnetic force, by a factor $\left[(\mathrm{m} / \mathrm{n})\left(\mathrm{R} / \mathrm{r}_{\mathrm{s}}\right)\right]^{2}$ for the toroidal rotation case. Equation (5) is therefore modified to

$$
\psi_{\mathrm{a}}=75 \mu \omega\left[\left(\mathrm{R} / \mathrm{r}_{\mathrm{s}}\right)(\mathrm{m} / \mathrm{n})\right]^{2}\left(\mathrm{~V}_{\mathrm{A}} \mathrm{w} / \mathrm{a}\right)^{-2} .
$$

Equation (6) implies that the NTM will be more easily locked for a lower plasma mass density, viscosity and mode frequency. A larger island width, minor radius of the rational surface and toroidal magnetic field also decrease the mode locking threshold. 
For ASDEX-Upgrade parameters with $B_{t}=2 T, a=0.5 m, R=1.7 m, n_{e}=5 \times 10^{19} \mathrm{~m}^{-3}, \mu$ $=0.5 \mathrm{~m}^{2} / \mathrm{s}, \mathrm{w}=0.1 \mathrm{a}, \mathrm{m}_{\mathrm{i}}=2 \mathrm{~m}_{\mathrm{p}}, \mathrm{r}_{3 / 2}=0.58 \mathrm{a}$, and $\mathrm{f}=\omega / 2 \pi=10 \mathrm{kHz}$, it is found from equation (6) that the mode locking of the $\mathrm{m} / \mathrm{n}=3 / 2$ mode occurs when

$$
\mathrm{b}_{\mathrm{rn}} \equiv \mathrm{b}_{\mathrm{ra}} / \mathrm{B}_{\mathrm{t}}=1.64 \times 10^{-3} .
$$

This value is larger than the intrinsic error field of ASDEX-Upgrade. The result is consistent with the experimental observation that 3/2 NTMs are not locked on ASDEX-Upgrade.

For a fusion reactor like ITER, assuming $B_{t}=6 T, a=2 m, R=6 m, f=1 \mathrm{kHz}$, and the other parameters as mentioned above, it is found that the $\mathrm{m} / \mathrm{n}=3 / 2$ mode is locked when

$$
\mathrm{b}_{\mathrm{rn}}=2.48 \times 10^{-5}
$$

For the $2 / 1$ mode, assuming $\mathrm{r}_{2 / 1}=0.75 \mathrm{a}$ and $\mathrm{f}=0.42 \mathrm{kHz}$ [19], one finds

$$
\mathrm{b}_{\mathrm{rn}}=4.38 \times 10^{-6} .
$$

where the relation $\psi_{\mathrm{m} / \mathrm{n}}(\mathrm{r}) \sim(\mathrm{r} / \mathrm{a})^{\mathrm{m}}$ is utilized.

It is seen that the low-m NTMs will be locked by a small error field in a fusion reactor. If the error field amplitude of the $m / n=2 / 1$ component is assumed to be $b_{r n}=10^{-4}$, the $2 / 1$ island will be locked for $\mathrm{w} \geq 0.021 \mathrm{a}$. When the effect of a resistive vacuum vessel is further included, the island will be locked at an even smaller width. Using an analytical model it was found that the $2 / 1$ island will be locked to the vacuum vessel for $w \geq 0.025 \mathrm{a}$ in ITER [19]. These results indicate that NTMs will stop rotation at a small amplitude in a fusion reactor, in contrast to the observations on existing tokamaks.

If plasma turbulence is absent around the rational surface, then the plasma viscosity is determined by the neoclassical transport, $\mu \approx 0.1 \mathrm{q}^{2} v_{\mathrm{i}} \rho_{\mathrm{i}}{ }^{2}$, where $\mathrm{q}$ is the safety factor, $v_{\mathrm{i}}$ is the ionion collision frequency, and $\rho_{i}$ is the ion Larmor radius [44]. For the ITER plasma with $T_{i}=10$ kev and other parameters as mentioned above, one finds $\mu=0.17 \times 10^{-3} \mathrm{~m}^{2} / \mathrm{s}\left(\mu \tau_{\mathrm{R}} / \mathrm{a}^{2}=0.024\right)$ at $r=r_{2 / 1}$. This value is more than three orders of magnitude lower than that used above $(\mu=0.5 \times$ $10^{-3} \mathrm{~m}^{2} / \mathrm{s}$ or $\mu \tau_{\mathrm{R}} / \mathrm{a}^{2}=70$ ), leading to a locking threshold to be approximately ten times smaller, when taking into account of the slow decrease of the locking threshold with decreasing 
plasma viscosity in the smaller $\mu$ regime as seen from figure 8 .

\section{Stabilization of NTMs by ECCD in the presence of a helical field}

Once the island is large enough to be locked at a particular toroidal and poloidal angle by the intrinsic machine error field, its o-point is not necessarily in the region covered by the RF waves deposition. In this case the RF current would have essentially no stabilizing effect $[22,24,25]$. In order to stabilize a large amplitude NTM by RF current for a reactor plasma, additional methods are needed either to maintain the island rotation or to control the island's phase required by RF wave deposition after mode locking.

For maintaining the island rotation one could use NBI as a momentum source, although NBI heating is not necessary for a burning plasma. Another possible way to rotate the island is to use a rotating helical field. Since the $2 / 1$ island can be locked by a small amplitude error field in a reactor plasma, an applied rotating helical field of a small amplitude would in turn be able to rotate the island.

A static helical field of an appropriate phase and amplitude can be used to ensure the island's o-point location to be in the RF wave deposition region, so that the RF current can still play a stabilizing role after the mode locking to the actively applied helical field. This issue is investigated in the following.

\subsection{Model for RF current}

To study the stabilization of NTMs by RF current in the presence of a static helical field, equations (2)-(4) are still used, but equation (1) is modified to

$$
\frac{d \psi}{d t}=E-\eta\left(j-j_{b}-j_{d}\right)
$$

where the RF driven current density $j_{\mathrm{d}}$ is included into Ohm's law [24,25].

Due to the spacial diffusion of fast electrons, the RF current density depends on both the wave deposition region and the transport of the fast electrons generated by the RF wave. 
An appropriate model for describing the RF current density profile has to include the RF current source as well as the parallel and the perpendicular transport of the fast electrons. Following reference [25] the fast electron density is described by the equation

$$
\frac{\partial n_{f}}{\partial t}=\nabla_{\|}\left(\chi_{\|} \nabla n_{f}\right)+\nabla_{\perp}\left(\chi_{\perp} \nabla n_{f}\right)+v_{f}\left(n_{f s}-n_{f}\right)
$$

where $\mathrm{n}_{\mathrm{f}}, \mathrm{v}_{\mathrm{f}}^{-1}, \chi_{\| \mathrm{f}}$ and $\chi_{\perp \mathrm{f}}$ are the density, the slowing down time, the parallel and perpendicular transport coefficients of the fast electrons. $\mathrm{n}_{\mathrm{fs}}$ is the fast electron source due to the RF waves given by $[24,25]$

$$
n_{f s}=n_{f s 0} \exp \left[-\left(\frac{r-r_{d s}}{w_{d s}}\right)^{2}\right] \Pi\left(h_{0}\right)
$$

where $\mathrm{n}_{\mathrm{fs} 0}, \mathrm{w}_{\mathrm{ds}}$ and $\mathrm{r}_{\mathrm{ds}}$ specify the magnitude, the radial half-width and the deposition radius of the source, respectively.

$\Pi\left(\mathrm{h}_{0}\right)$ in equation (10) is a square box function for describing the instantaneous wave deposition along the helical angle $\mathrm{h}=\mathrm{m} \theta+\mathrm{n} \phi$ [24,25], being defined as $\Pi\left(\mathrm{h}_{0}\right)=1$ for $\mathrm{h}_{\mathrm{on}}<\mathrm{h}_{0}<\mathrm{h}_{\text {off }}$, and $\Pi\left(\mathrm{h}_{0}\right)=0$ elsewhere. $\mathrm{h}_{0}$ is the instantaneous helical angle of the island's opoint, and $h_{\text {on }}\left(h_{\text {off }}\right)$ is the helical angle at which the RF wave is turned on (off). The wave deposition region is chosen to be centered at $\mathrm{h}=0$ with a width $\Delta \mathrm{h}$ along the helical angle, and $\mathrm{h}_{\mathrm{on}}=-\pi / 2$ and $\mathrm{h}_{\text {off }}=\pi / 2$ are used. This leads to $\Pi\left(\mathrm{h}_{0}\right)=1\left(\Pi\left(\mathrm{h}_{0}\right)=0\right)$ when the island's o-point ( $\mathrm{x}-$ point) is close to $\mathrm{h}=0$, corresponding to modulated current drive (MCD) for a rotating island, being more effective for stabilizing rotating NTMs than continuous current drive if the radial RF wave deposition width is larger than the island width $[22,45]$. The phase of the applied helical field is chosen to lock the island's o-point at $\mathrm{h}=0$, which allows the $\mathrm{RF}$ current to be around the island's o-point to stabilize the NTM after mode locking.

Assuming that the driven current density is proportional to the fast electron density, $\mathrm{j}_{\mathrm{d}} \sim \mathrm{n}_{\mathrm{f}}$, the total driven current $\mathrm{I}_{\mathrm{d}}$ is obtained by integrating $\mathrm{j}_{\mathrm{d}}$ over the plasma cross section. Similarly, the RF source current, $\mathrm{I}_{\mathrm{ds}}$, is obtained by assuming the source current density 
$\mathrm{j}_{\mathrm{ds}} \sim \mathrm{n}_{\mathrm{fs} 0}$ and integrating $\mathrm{j}_{\mathrm{ds}}$ over the plasma cross section. The half width $\mathrm{w}_{\mathrm{ds}}$ and the total $\mathrm{RF}$ source current $\mathrm{I}_{\mathrm{ds}}$ can be given by a ray-tracing code and a Fokker-Planck code [24].

\subsection{Numerical results}

The time evolution of the $m / n=3 / 2$ NTM frequency is shown in figure 11 for three cases, $b_{\mathrm{rn}}=0,9 \times 10^{-7}$, and $3 \times 10^{-6}$, respectively. The input parameters are as follows: $\mathrm{f}_{\mathrm{b}}=0.063$, $\mathrm{I}_{\mathrm{ds}} / \mathrm{I}_{\mathrm{P}}=0.03, \mathrm{w}_{\mathrm{ds}} / \mathrm{a}=0.1, \Delta \mathrm{h}=0.482, \mathrm{v}_{\mathrm{f}}=4 \times 10^{3} / \tau_{\mathrm{R}}, \chi_{\perp \mathrm{f}}=1.0 \mathrm{a}^{2} / \tau_{\mathrm{R}}$, and $\chi_{\| f} / \chi_{\perp \mathrm{f}}=10^{10}$, where $\mathrm{I}_{\mathrm{P}}$ is the total plasma current. The minor radius of the RF current density peak, $\mathrm{r}_{\mathrm{ds}}$, is chosen to be at the $\mathrm{q}=3 / 2$ rational surface $\mathrm{r}_{\mathrm{s}}$. The other parameters are the same as those for figure 1 . For vanishing error field $\left(b_{\mathrm{rn}}=0\right)$ the normalized mode frequency $\omega \tau_{\mathrm{R}}=-1.2 \times 10^{4}$, being only determined by the equilibrium plasma rotation. For a small $b_{r n}\left(b_{r n}=9 \times 10^{-7}\right)$, the mode frequency oscillates around the equilibrium value. A further increase in $b_{\mathrm{rn}}\left(b_{\mathrm{rn}}=3 \times 10^{-6}\right)$ leads to mode locking to the applied static helical field.

For the parameters used in figure 11, the time evolution of the normalized RF current, $I_{d} / I_{p}$, is shown in figure 12 for $b_{r n}=0,9 \times 10^{-7}$, and $3 \times 10^{-6}$, respectively. The RF current $I_{d}$ rises in a time scale of $v_{f}^{-1}$ and oscillates afterwards around $0.5 \mathrm{I}_{\mathrm{ds}}$ for $\mathrm{b}_{\mathrm{rn}}=0$ and $9 \times 10^{-7}$. In these two cases the island is rotating, and the source term in equation (10) is turned on for half the time only when the island's o-point is close to the RF wave deposition region around $\mathrm{h}=0$.

For a sufficiently large helical field $\left(\mathrm{b}_{\mathrm{rn}}=3 \times 10^{-6}\right)$, the island's o-point is locked by the field at $h=0$, so that the RF current is always turned on, leading to $I_{d}=I_{d s}$.

The corresponding time evolution of the normalized island width w/a is shown in figure 13 for $b_{\mathrm{rn}}=0,9 \times 10^{-7}$, and $3 \times 10^{-6}$, respectively. The saturated island width increases a little for a small $b_{r n}\left(b_{r n}=9 \times 10^{-7}\right)$ compared to the case with $b_{r n}=0$. For a sufficiently larger $b_{r n}$ $\left(b_{\mathrm{rn}}=3 \times 10^{-6}\right)$, however, the saturated island width is significantly smaller than that obtained with $b_{r n}=0$, showing an enhanced stabilizing effect of the RF current in this case. This is due to the two times larger $I_{d}$ for the case $b_{r n}=3 \times 10^{-6}$ as seen in figure 12 , so that the stabilizing 
effect by the RF current is larger.

To compare the destabilizing effect of the helical field to the increased stabilizing effect due to a larger RF current, the saturated island width is shown as a function of $b_{r n}$ in figure 14 for two cases. The first case shown by the solid line with squares is obtained with $\mathrm{f}_{\mathrm{b}}=0.063, \mathrm{I}_{\mathrm{ds}}=0.03$ and $\mathrm{w}_{\mathrm{ds}}=0.1 \mathrm{a}$. The straight dotted line shows the corresponding saturated island width $\mathrm{w}=0.048 \mathrm{a}$ obtained for $\mathrm{b}_{\mathrm{rn}}=0$. It is seen that the island width is smaller than that obtained without the helical field in the region $10^{-6}<\mathrm{b}_{\mathrm{rn}}<4.5 \times 10^{-4}$. In this region the island is locked by the helical field, so that the stabilizing effect of the RF current is larger due to a larger RF current. For $b_{\mathrm{rn}}>4.5 \times 10^{-4}$, the destabilizing effect of the helical field is too strong, leading to a larger island width than that obtained with $b_{r n}=0$. The second case shown by the solid line with circles is obtained for $\mathrm{f}_{\mathrm{b}}=0.19, \mathrm{I}_{\mathrm{d}}=0.02$ and $\mathrm{w}_{\mathrm{ds}}=0.04 \mathrm{a}$. The straight dashed line shows the corresponding saturated island width $\mathrm{w}=0.074 \mathrm{a}$ obtained for $\mathrm{b}_{\mathrm{rn}}=0$. The island width is smaller than that obtained without the helical field in the region $10^{-6}<\mathrm{b}_{\mathrm{rn}}<1.8 \times 10^{-3}$. The upper limit of the enhanced stabilization region extends to a larger value of $b_{\mathrm{rn}}$ compared to the first case, since the helical field is less important in determining the island width for a higher fraction of the bootstrap current density. The increased stabilizing effect is nearly fully reached for $10^{-5}<b_{\mathrm{rn}}<10^{-4}$, and such a magnitude of $b_{\mathrm{rn}}$ is able to lock the $3 / 2$ and the $2 / 1$ NTM rotating in the toroidal direction in a fusion reactor as seen from Section 2.

\subsection{Discussion on NTM stabilization by RF current}

For $m / n=2 / 1$ NTMs, similar results to those shown in figure 14 are obtained. Above results show that despite the destabilizing effect of the helical field, by choosing an optimum phase of the island with respect to the RF current, the overall effect can be stabilizing due to the two times larger RF current around the island's o-point. These results indicate that the helical field can not only be used to adjust the island phase after mode locking to the intrinsic machine error field, it can also be actively utilized to increase the stabilizing efficiency of NTMs by RF current. Such a method is expected to be useful for stabilizing large amplitude 
NTMs, especially the 2/1 NTM, in plasmas with a high bootstrap current fraction. A possible application of this method is the avoidance of disruptions caused by large locked islands.

If the helical field causes a decrease in the frequency of a rotating NTM such that the rotation period is longer compared to the slowing down time of the fast electrons, a larger stabilizing effect on a rotating NTM by the RF current is also expected [24,25].

\section{Summary}

The mode locking of NTMs and its stabilization by RF current is investigated in the present paper using large aspect-ratio tokamak approximation. The toroidal mode coupling and Glasser effect are neglected under this approximation. These effects are however not expected to be significant if the considered mode is dominant [6]. Future work to use a full toroidal geometry would allow to further take into account these effects and to give a more precise mode locking threshold. In addition, using an equilibrium toroidal plasma rotation rather than a poloidal one would be helpful to compare with equation (6). Only single helicity is used in our calculation. This holds if the amplitude of the principal mode is much larger than that of other modes, while the error field amplitude of the corresponding helicity is larger than or comparable to that of other helicities. The ion polarization current associated with the diamagnetic drift is neglected, being valid for a sufficiently large island that the radial electron pressure profile is flattened across the island [43]. The effect of the plasma beta value on the mode locking is implicitly included by the island width. The saturated island width is larger for a higher fraction of bootstrap current density fraction at the rational surface or a higher beta value, so that the corresponding locking threshold is lower. To our knowledge, the exact value of plasma viscosity of a reactor plasma is not known, although the viscosity is expected to be at the level of the anomalous perpendicular heat conductivity. This leads to somewhat uncertainty in predicting the precise locking threshold of a fusion reactor.

In summary, the locking of neoclassical tearing modes by an error field is studied by numerical modelling. It is shown that for a low mode frequency and a large plasma viscosity, 
the mode locking threshold is proportional to the plasma viscosity and mode frequency but inversely proportional to the square of the Alfven velocity and the island width. Such a scaling is the same as that of the classical tearing mode. The low-m NTMs is expected to be locked by a small amplitude error field in a reactor plasma.

The stabilization of NTMs by RF current in the presence of an applied static helical field is therefore further investigated. It is found that an actively applied field for controlling the island phase can enhance the stabilizing effect of the RF current. Such a method is expected to be useful for the stabilization of large amplitude NTMs, especially $\mathrm{m} / \mathrm{n}=2 / 1$ NTMs, in a fusion reactor. 


\section{References}

[1] Z. Chang, J. D. Callen, E. D. Fredrickson, et al., Phys. Rev. Letts, 74, 4663(1995).

[2] H. Zohm, G. Gantenbein, A. Gude, S. Günter, et al., Nucl. Fusion 41, 197(2001).

[3] S. Günter,A. Gude, M. Maraschek, et al., Phys. Rev. Letters, 87, 275001(2001).

[4] R. J. La Haye, L. L. Lao, E. J. Strait, and T. S. Taylor, Nucl. Fusion 37, 397(1997).

[5] S. Günter, A. Gude, M. Maraschek, et al., Plasma Phys. Control. Fusion, 41, 767(1999).

[6] O. Sauter, R.J. La Haye, Z. Chang, D.A. Gates, et al., Phys. Plasmas 4, 1654(1997).

[7] D. A. Gates, B. Lloyd, A. W. Morris, et al., Nucl. Fusion 37, 1593(1997).

[8] S. Günter, A. Gude, M. Marschek, et al., Nucl. Fusion 38, 1431(1998).

[9] A. I. Smolyakov, Plasma Phys. Control. Fusion, 35, 657(1993).

[10] H. R. Wilson, J.W. Connor, R.J. Hastie, and C. C. Hegna, Phys. Plasmas 3, 248(1996).

[11] Richard Fitzpatrick, Phys. Plasmas 2, 825(1995).

[12] M. Zabiego and J. D. Callen, Nucl. Fusion 37, 361(1997).

[13] A. Gude, S. Günter, S. Sesnic, ASDEX Upgrade Team, Nucl. Fusion 39, 127(1999).

[14] J.W. Connor, F. L. Waelbroeck, and H. R. Wilson, Phys. Plasmas 8, 2835(2001).

[15] Q. Yu, S. Günter and Bruce D. Scott, Phys. Plasmas 10, 797(2003).

[16] R. J. La Haye, R. J. Buttery, et al., Phys. Plasmas 7, 3349(2000).

[17] H. Zohm, G. Gantenbein, G. Giruzzi, S. Günter, et al., Nucl. Fusion 39, 577(1999).

[18] G. Gantenbein, H. Zohm, G. Giruzzi, S. Günter, et al, Phys. Rev. Letts, 85, 1242(2000).

[19] R.J. La Haye, R. Prater, R.J. Buttery, et al, Nucl. Fusion 46, 451 (2006).

[20] H. Zohm, G. Gantenbein, F. Leuterer, A. Manini, et al., Nucl. Fusion 47, 228(2007).

[21] A. Isayama, Y. Kamada, S. Ide, et al., Plasma Phys. Control. Fusion 42, L37(2000).

[22] M. Maraschek, G. Gantenbein, Q. Yu, et al, Phys. Rev. Letts, 98025005 (2007).

[23] C. C. Hegna and J. D. Callen, Phys. Plasmas 4, 2940(1997).

[24] G. Giruzzi, M. Zabiego, T. A. Gianakon, et al., Nucl. Fusion 39, 107(1999).

[25] Q. Yu, S. Günter, G. Giruzzi, K. Lackner, and M. Zabiego, Phys. Plasmas 7, 312(2000). 
[26] F. W. Perkins, R. W. Haevey, M. Makowski, and M.N. Rosenbluth, in Proceedings of the 24th EPS Conference on Controlled Fusion and Plasma Physics (European Physical Society), 9-13 June 1997, Berchtesgaden, Germany, Vol. 3, p. 1017.

[27] M.F.F. Nave and J.A. Wesson, Nucl. Fusion 30, 2575 (1990).

[28] T.C. Hender, R. Fitzpatrick, A.W. Morris, et al, Nucl. Fusion 32, 2091 (1992).

[29] H. Zohm, A. Kallenbach, H. Bruhns, et al, Europhys. Lett. 11, 745 (1990).

[30] R. Fitzpatrick and T.C. Hender, Phys. Fluids B 3, 644 (1991).

[31] R. Fitzpatrick, Nucl. Fusion 33, 1049 (1993).

[32] R. Fitzpatrick, Phys. Plasmas 5, 3325 (1998).

[33] R.J. Buttery, et al, Nucl. Fusion 40, 807 (2000).

[34] R.J. Buttery, et al, Nucl. Fusion 39, 1827 (1999).

[35] R.J. La Haye, R. Fitzpatrick, T.C. Hender et al., Phys. Fluids B 4, 2098 (1992).

[36] R.J. La Haye, A.W. Hyatt and J.T. Scoville, Nucl. Fusion 32, 2119 (1992).

[37] S.W. Wolfe, I.H. Hutchinson, R.S. Granetz et al., Phys. Plasmas 12, 056110 (2005).

[38] H.R. Koslowski, Y. Liang A. Krämer-Flecken et al., Nucl. Fusion 46, L1 (2006).

[39] K.H. Finken, S.S. Abdullaev, et al., Phys. Rev. Letts, 94, 015003(2005).

[40] Q. Yu, S. Günter, Y. Kikuchi, and K. H. Finken, Nuclear Fusion 48, 024007 (2008).

[41] Q. Yu, S. Günter and K. Lackner, Phys. Plasmas 11, 140(2004).

[42] S. Günter, Q. Yu, J. Krüger, and K. Lackner, J. Comp. Phys. 209, 354 (2005)

[43] Q. Yu, Phys. Plasmas 13062310 (2006)

[44] F.L. Hinton and S.K. Wong, Phys. Fluids 28, 3082 (1985).

[45] Q. Yu, X.D. Zhang, and S. Günter, Phys. Plasmas 11, 1960 (2004). 


\section{CAPTION}

Figure 1 Normalized island width w/a versus the normalized time $t / \tau_{R}$. The solid (dashed) curve corresponds to an error field amplitude of $\psi_{\mathrm{a}}=5 \times 10^{-8}\left(\psi_{\mathrm{a}}=10^{-7}\right)$.

Figure 2 Corresponding to figure 1 , time evolution of the mode frequency for $\psi_{\mathrm{a}}=5 \times 10^{-8}$ (solid curve) and $10^{-7}$ (dashed). For $\psi_{\mathrm{a}}=5 \times 10^{-8}$ the mode frequency oscillates around a finite value. For $\psi_{\mathrm{a}}=10^{-7}$ the island is locked by the error field.

Figure 3 The radial profile of $\psi_{3 / 2}$ in steady state for $\psi_{\mathrm{a}}=10^{-7}$. The solid (dashed) curve represents the real (imaginary) part. The smaller imaginary part (compared to the real one) indicates a small phase shift between the mode and the error field.

Figure 4 Radial profiles of the $m / n=0 / 0$ component of the poloidal plasma velocity in steady state for $\psi_{\mathrm{a}}=5 \times 10^{-8}$ (solid) and $10^{-7}$ (dotted). The dashed curve shows the original equilibrium profile. For $\psi_{\mathrm{a}}=5 \times 10^{-8}$ the local velocity around the rational surface oscillates as shown by the two solid curves for $\mathrm{t}=0.09680$ and $0.09681 \tau_{\mathrm{R}}$, respectively.

Figure 5 The radial profile of the $m / n=0 / 0$ component electron temperature in steady state for $\psi_{\mathrm{a}}=10^{-7}$. The dotted curve is the original equilibrium one. The local temperature gradient around the rational surface reduces to nearly zero.

Figure 6 (color online) The required $\psi_{\mathrm{a}}$ for mode locking versus the magnetic Reynolds number S. The solid circles (empty squares) show the $\psi_{\mathrm{a}}$ for which the mode is locked (not locked). The mode locking threshold is proportional to $\mathrm{S}^{-2}$.

Figure 7 (color online) The required $\psi_{\mathrm{a}}$ for mode locking versus the normalized island width w/a. The solid circles (empty squares) show the $\psi_{\mathrm{a}}$ for which the mode is locked (not locked). The mode locking threshold is proportional to $\mathrm{w}^{-2}$.

Figure $8 \quad$ (color online) The required $\psi_{\mathrm{a}}$ for mode locking versus plasma viscosity $\mu$ (in the unit $\mathrm{a}^{2} / \tau_{\mathrm{R}}$ ). The solid circles (empty squares) show the $\psi_{\mathrm{a}}$ for which the mode is locked (not locked). The mode locking threshold is proportional to $\mu$ for large $\mu$, but the dependence 
on $\mu$ is weak for small $\mu$.

Figure 9 (color online) The required $\psi_{\mathrm{a}}$ for mode locking versus the normalized island angular rotation frequency $\omega \tau_{R}$ for two cases, $\tau_{\mu}=0.1 \tau_{R}$ and $\tau_{\mu}=10^{-4} \tau_{R}$. The solid circles (empty squares) show the $\psi_{\mathrm{a}}$ for which the mode is locked (not locked). The mode locking threshold is linearly proportional to $\omega$ for small $\tau_{\mu}$ or $\omega$.

Figure 10 (color online) The required $\psi_{\mathrm{a}}$ for mode locking versus $\omega \tau_{\mathrm{R}}$ for the $2 / 1$ mode for three cases: $\mathrm{f}_{\mathrm{b}}=0$ and 0.052 for $\tau_{\mu}=10^{-4} \tau_{\mathrm{R}}$, and $\mathrm{f}_{\mathrm{b}}=0.052$ for $\tau_{\mu}=10^{-2} \tau_{\mathrm{R}}$. The solid circles (empty squares) show the $\psi_{\mathrm{a}}$ for which the mode is locked (not locked). The results are similar to that of the $3 / 2$ mode.

Figure 11 The time evolution of the $m / n=3 / 2$ NTM frequency for $b_{r n}=0,9 \times 10^{-7}$ and $3 \times$ $10^{-6}$, with $\mathrm{I}_{\mathrm{ds}} / \mathrm{I}_{\mathrm{p}}=0.03, \mathrm{w}_{\mathrm{ds}} / \mathrm{a}=0.1, \chi_{\perp \mathrm{f}}=1.0 \mathrm{a}^{2} / \tau_{\mathrm{R}}, \Delta \mathrm{h}=0.482, \mathrm{v}_{\mathrm{f}}=4 \times 10^{3} / \tau_{\mathrm{R}}, \chi_{\| \mathrm{If}} / \chi_{\perp \mathrm{f}}=10^{10}, \mathrm{r}_{\mathrm{ds}}=\mathrm{r}_{\mathrm{s}}$, and $\mathrm{f}_{\mathrm{b}}$ $=0.063$. The other parameters are the same as those for figure 1 .

Figure 12 Corresponding to figure 11, the time evolution of the normalized RF current, $I_{d} / I_{p}$, for $b_{r n}=0,9 \times 10^{-7}$ and $3 \times 10^{-6}$. $I_{d}$ rises in a time scale of $v_{f}^{-1}$ and oscillates later around $0.5 I_{d s}$ for $b_{r n}=0$ and $9 \times 10^{-7}$. For $b_{r n}=3 \times 10^{-6}$, the island's o-point is locked at $h=0$, so that $I_{d}=I_{d s}$. Figure 13 Corresponding to figure 11, the time evolution of the normalized island width w/a for $b_{\mathrm{rn}}=0,9 \times 10^{-7}$ and $3 \times 10^{-6}$. For $b_{\mathrm{rn}}=3 \times 10^{-6}$, the island width is significantly smaller than that obtained with $b_{\mathrm{rn}}=0$, showing an increased stabilizing effect of the RF current. Figure 14 The saturated island width versus $\log \left(b_{\mathrm{rn}}\right)$. The line with squares (circles) corresponds to $\mathrm{f}_{\mathrm{b}}=0.063, \mathrm{I}_{\mathrm{ds}}=0.03$ and $\mathrm{w}_{\mathrm{ds}}=0.1 \mathrm{a}\left(\mathrm{f}_{\mathrm{b}}=0.19, \mathrm{I}_{\mathrm{ds}}=0.02\right.$ and $\left.\mathrm{w}_{\mathrm{ds}}=0.04 \mathrm{a}\right)$. The straight dotted and dashed lines show the corresponding island widths for $b_{\mathrm{rn}}=0$. The island width is smaller than that obtained with $b_{r n}=0$ for a certain range of $b_{r n}$, up to $b_{r n}<1.8 \times 10^{-3}$ for $f_{b}=0.19$. 


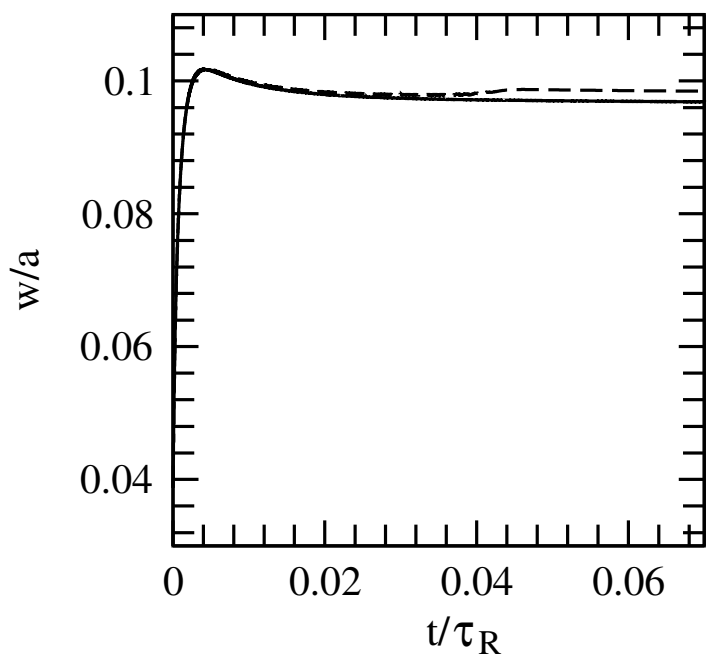

Figure 1 


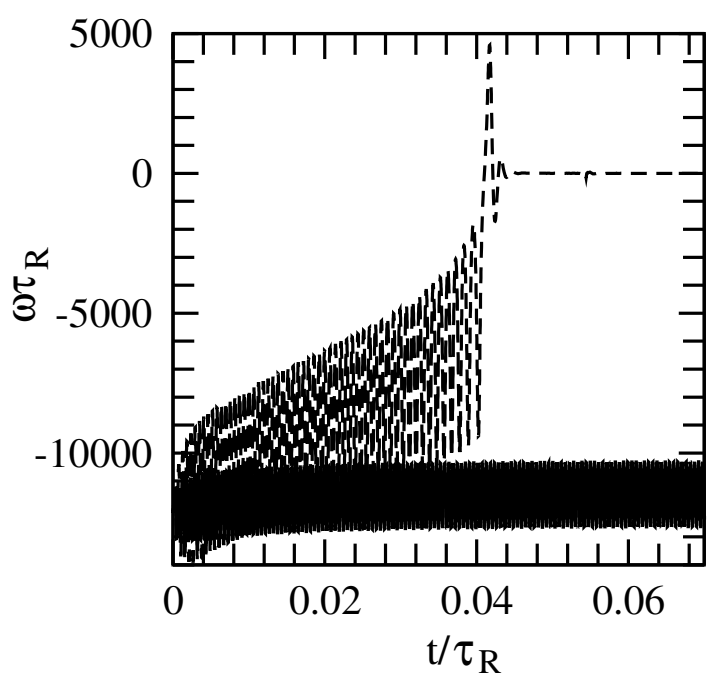

Figure 2 


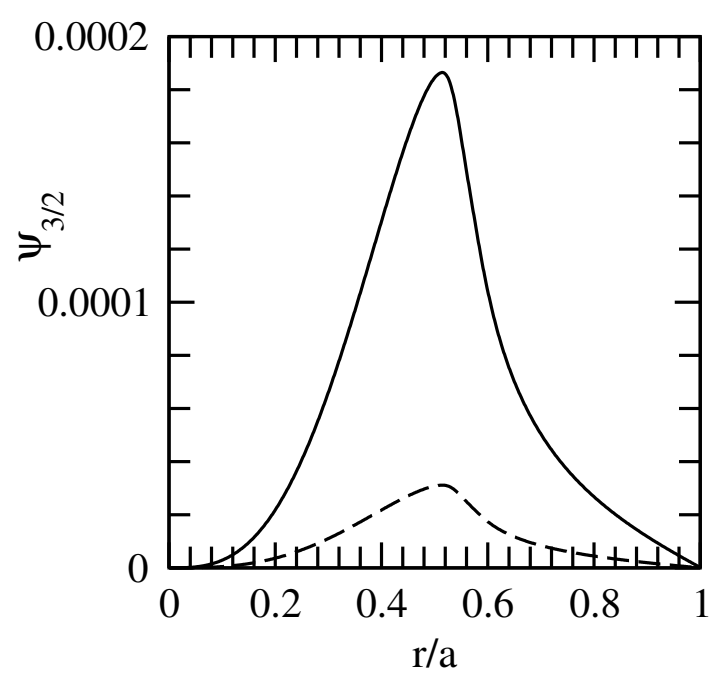

Figure 3 


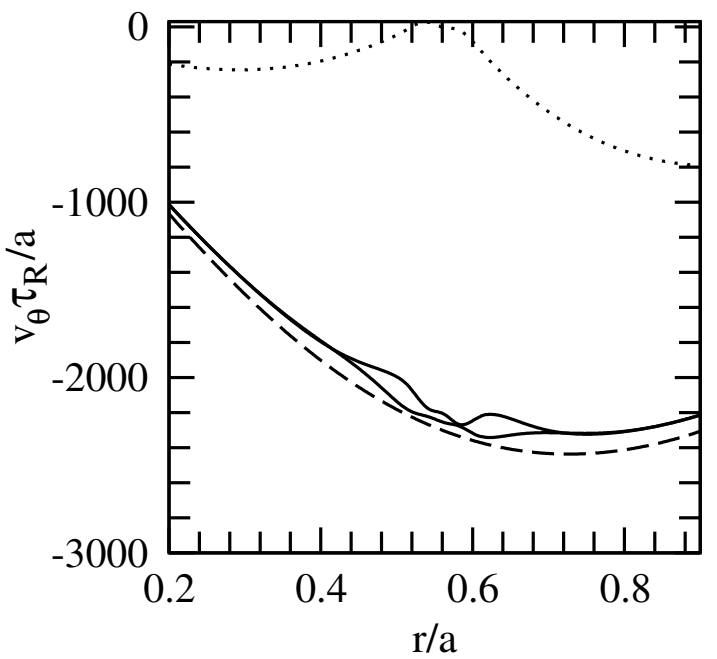

Figure 4 


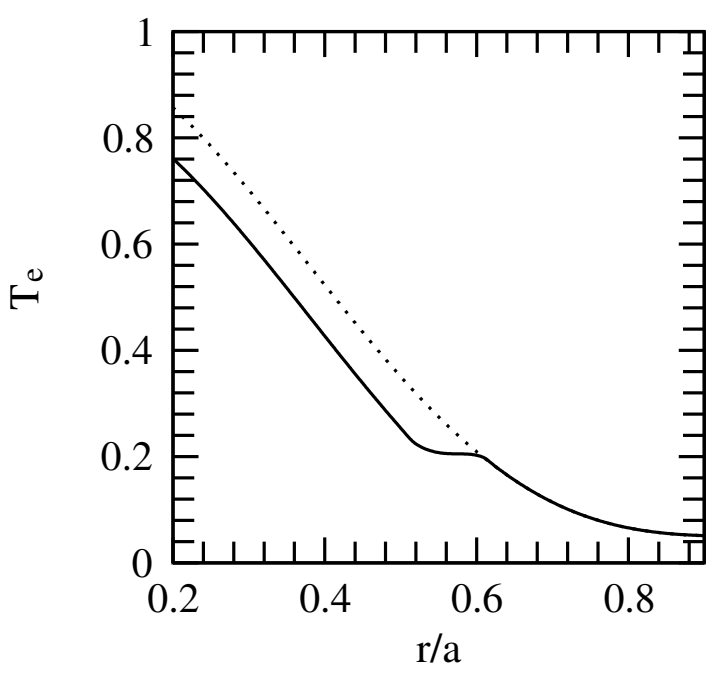

Figure 5 


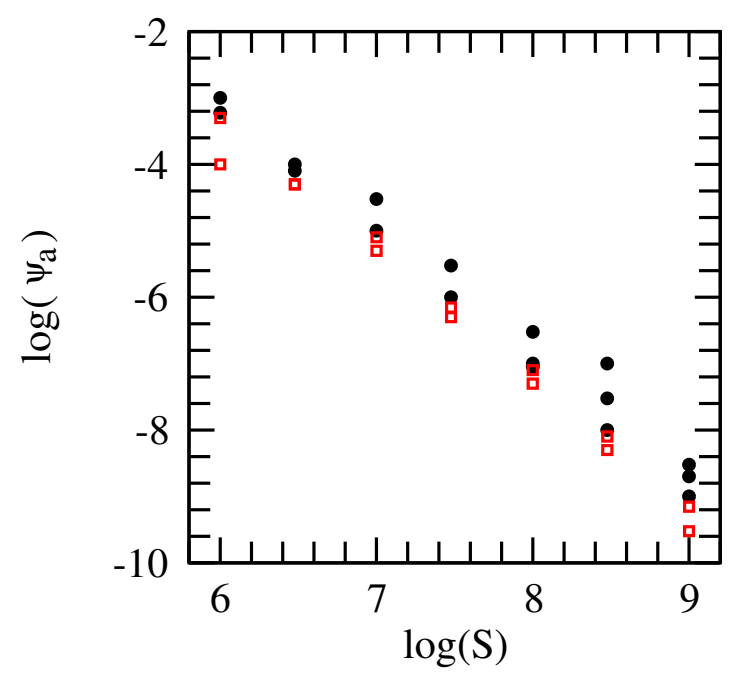

Figure 6 


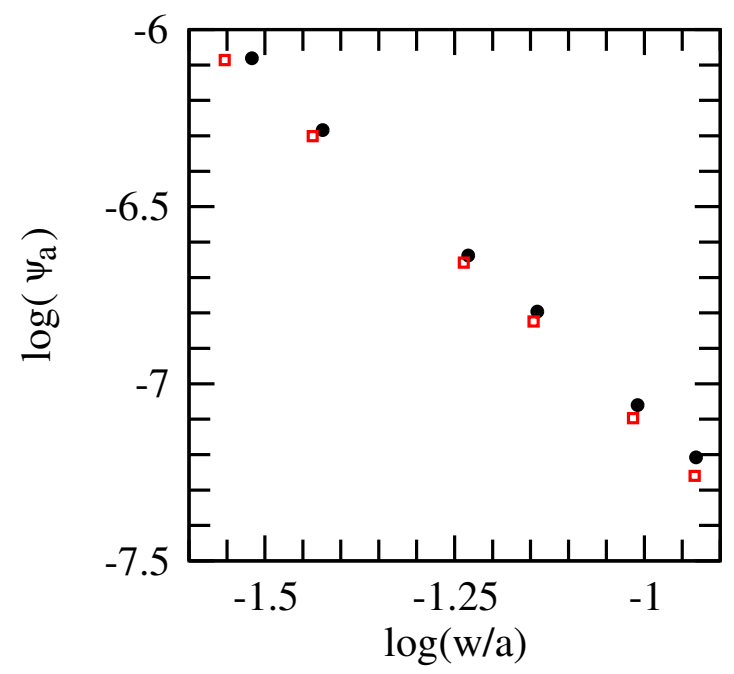

Figure 7 


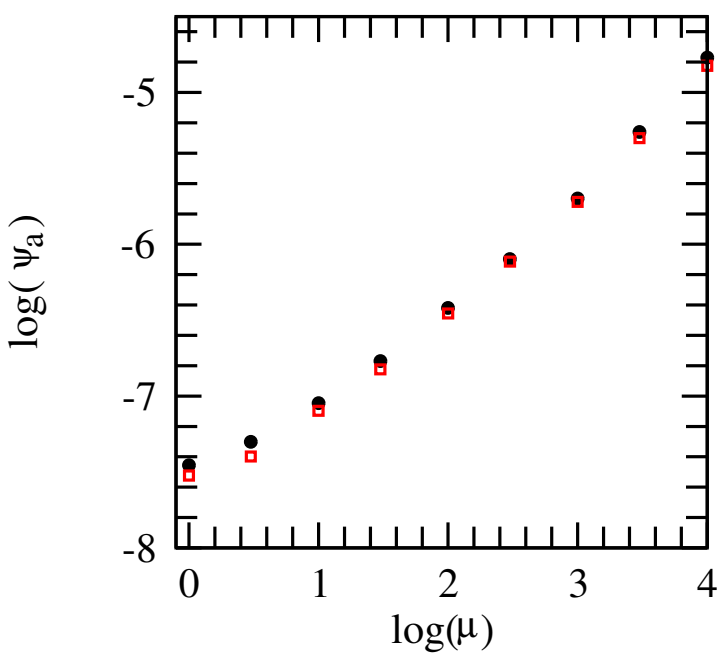

Figure 8 


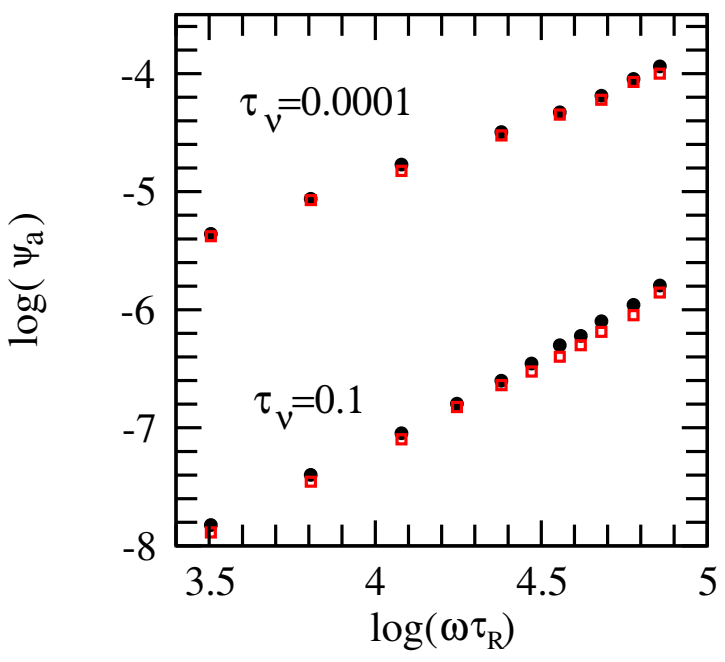

Figure 9 


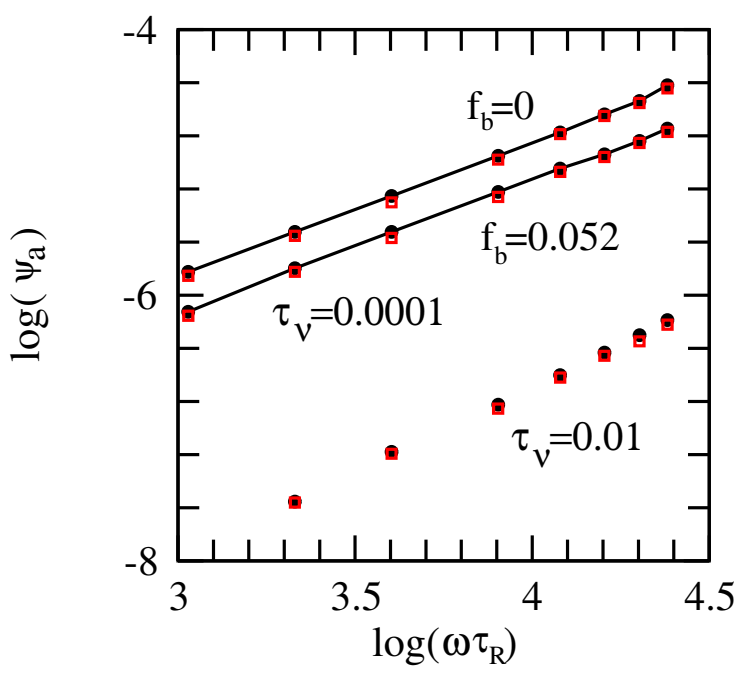

Figure 10 


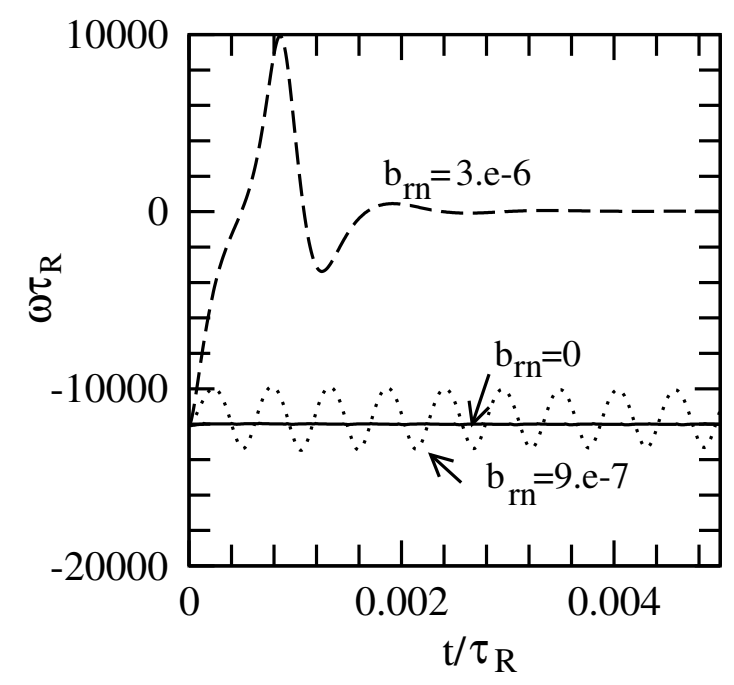

Figure 11 


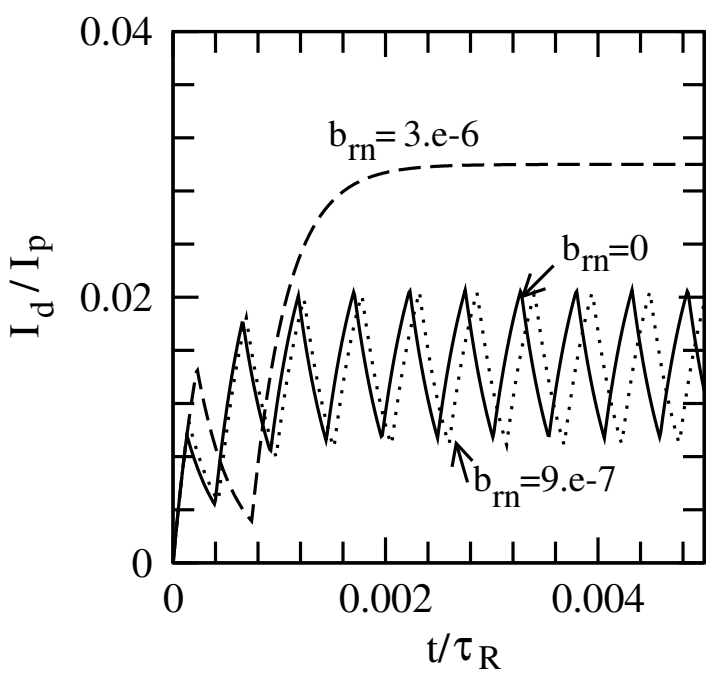

Figure 12 


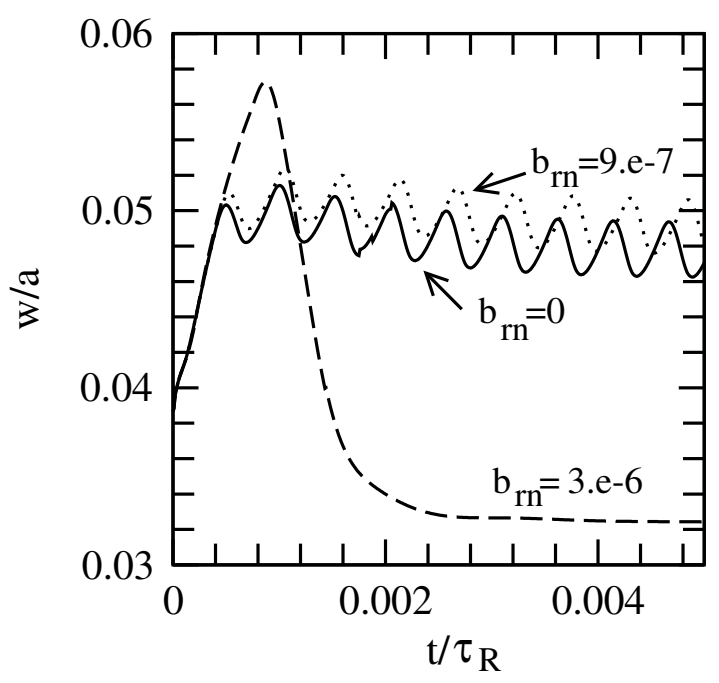

Figure 13 


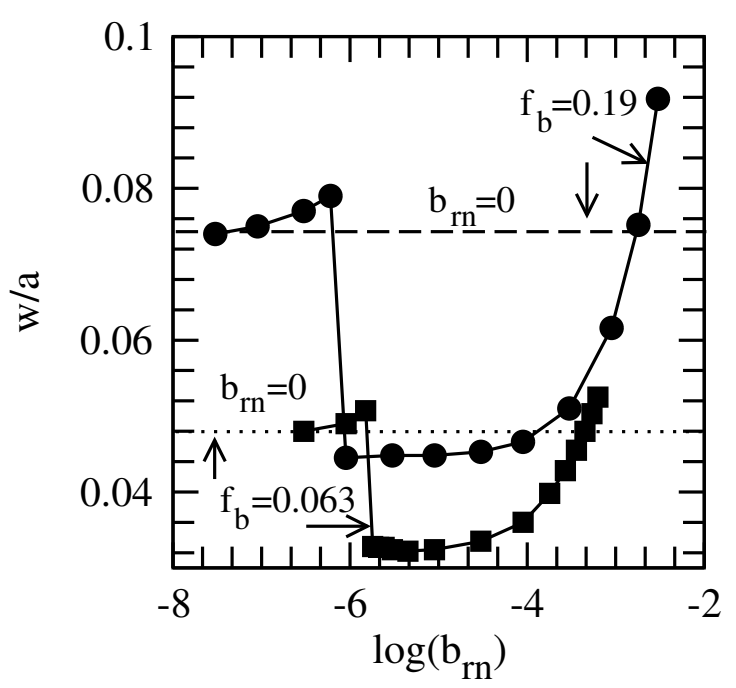

Figure 14 